\title{
Controlled growth of one-dimensional clusters of molybdenum atoms using double-walled carbon nanotube templating
}

\author{
Yousheng Tao, ${ }^{1, a)}$ Hiroyuki Muramatsu, ${ }^{1}$ Takuya Hayashi, ${ }^{2}$ Yoong Ahm Kim, ${ }^{2}$ \\ Daisuke Shimamoto, ${ }^{2}$ Morinobu Endo, ${ }^{1,2}$ Katsumi Kaneko, ${ }^{3}$ Mauricio Terrones, ${ }^{4}$ and \\ Mildred S. Dresselhaus ${ }^{5}$ \\ ${ }^{1}$ Institute of Carbon Science and Technology, Shinshu University, Nagano 380-8553, Japan \\ ${ }^{2}$ Faculty of Engineering, Shinshu University, Nagano 380-8553, Japan \\ ${ }^{3}$ Department of Chemistry, Chiba University, Chiba 263-8522, Japan \\ ${ }^{4}$ Department of Advanced Materials, IPICYT, Camino a La Presa San José 2055, Col. Lomas $4^{a}$. Sección, \\ San Luis Potosi 7816, Mexico \\ ${ }^{5}$ Massachusetts Institute of Technology, Cambridge, Massachusetts 02139-4307, USA
}

(Received 27 November 2008; accepted 2 February 2009; published online 17 March 2009)

\begin{abstract}
We report the controlled growth of one-dimensional clusters of molybdenum atoms inside the inner cores of double-walled carbon nanotubes. A combined characterization including high resolution transmission electron microscopy, nitrogen adsorption measurement at $77 \mathrm{~K}$, x-ray photoelectron spectroscopy, Raman spectroscopy, and thermogravimetric analysis reveals that the growth of one-dimensional Mo clusters can be controlled by varying the reaction conditions. The products have specific surface areas of $360-480 \mathrm{~m}^{2} \mathrm{~g}^{-1}$, and their characteristic properties are attributed to the presence of Mo cluster, which affect the electronic structure and can be exploited for the development of nanotube electronic devices. (c) 2009 American Institute of Physics.
\end{abstract}

[DOI: $10.1063 / 1.3089576$ ]

The discovery and identification of carbon nanotubes have stimulated scientific and industrial interest due to their outstanding mechanical and electronic properties and potential applications. Recently a unique class of hybrid materials in the form of assembled atomic or cluster chains inside carbon nanotubes suggested to have electronic and mechanical properties that differ from those of empty carbon nanotubes, thus there is a strong interest in developing an efficient fabrication technique so that these filled nanotubes could be used in nanoelectronic and nano-optoelectronic devices. ${ }^{1}$ Since $\mathrm{C}_{60}$ molecules have been successfully introduced inside single wall carbon nanotubes (SWNTs), often called as peapods, ${ }^{2}$ efforts have recently been made in order to improve the yields of such fullerene encapsulated SWNTs. A step made forward corresponded to the insertion of gadolinium encapsulated $\mathrm{C}_{82}$ in SWNTs, thus forming a peapodlike structure. ${ }^{3}$ Compared with empty one, the SWNT encapsulated with gadolinium endohedral metallofullerenes showed much larger temperature dependence on electrical resistance. At present, state-of-the-art oxygen-bridged gadolinium (III) clusters of many metal atoms have been reported to be selectively deposited in nanowindows of carbon nanohorns. ${ }^{4}$ However, the highly expected one-dimensional metallic atomic wires are still difficult to obtain using this fabrication technique because the metal atoms tend to form large metal clusters. ${ }^{5}$ Muramatsu et al. have found atomic wires that could be inserted in the inner nanotube of doublewalled carbon nanotubes (DWNTs). ${ }^{6}$ A further theoreticalexperimental study has indicated that these wires consist of half a bcc-Mo cell width nanowire. ${ }^{7}$ Interestingly, these Mo wires induce a metallic behavior within semiconducting nanotubes. Along this line, here we report that the growth of

${ }^{\text {a)}}$ Electronic mail: tao@endomoribu.shinshu-u.ac.jp. one-dimensional clusters of Mo atoms can be efficiently controlled by varying the reaction conditions.

DWNTs were prepared using a catalytic chemical vapor deposition method and an optimized two-step postsynthesis purification procedure, in which the iron catalysts and the magnesium oxide carrier were removed by hydrochloric acid and carbon impurities were removed by oxidation. ${ }^{8} \mathrm{We}$ prepared two samples from DWNTs, hexaammanium heptamolybdate tetrahydrate (minimum $99.0 \%$ ), hydrochloric acid (35.0\%-37.0\%) and distilled water with composition (weight basis) 1:10:2750:2650 and 1:100:2750:2650, respectively. The samples were synthesized by impregnation of DWNTs in the mixed solutions and then annealed in air at $773 \mathrm{~K}$ for $1 \mathrm{~h}$. The sample synthesized from the low Mo ratio is named as LMo-DWNTs, and another from the high Mo ratio is named as HMo-DWNTs. High-resolution transmission electron micrographs (HRTEMs) of samples were obtained on a JEOL JEM-2010FEF transmission electron microscope, operating at $200 \mathrm{keV}$. The nitrogen adsorption isotherms were measured at $77 \mathrm{~K}$ using a Quantachrome Autosorb-1 gas adsorption analyzer, after samples were evacuated at $10^{-4} \mathrm{~Pa}$ and $383 \mathrm{~K}$ for $2 \mathrm{~h}$. X-ray photoelectron spectroscopy (XPS) measurements were carried out using a Shimadzu ESCA3200 spectrometer at a base pressure of $10^{-6} \mathrm{~Pa}$ using $\mathrm{Mg} K \alpha(1253.6 \mathrm{eV})$ x rays. In order to compensate for a possible charging effect, binding energies were calibrated with respect to the position of the $\mathrm{C} 1 s$, this value being assumed constant at $284.5 \mathrm{eV}$. Raman spectra were obtained with a Kaiser Holo Laboratory 5000 system. Sample excitation was done with laser energies of $1.96 \mathrm{eV}(633 \mathrm{~nm} \mathrm{He}-\mathrm{Ne}$ laser line). The thermogravimetric (TG) analysis of the samples was performed on Rigaku Therm plus TG8120 at a heating rate of $5 \mathrm{~K} \mathrm{~min}^{-1}$ in an air flow of $200 \mathrm{ml} / \mathrm{min}$.

Figure 1 shows the HRTEM images of LMo-DWNTs and HMo-DWNTs. The isolated features in the interior of DWNTs as indicated by the solid arrows were Mo atoms that 


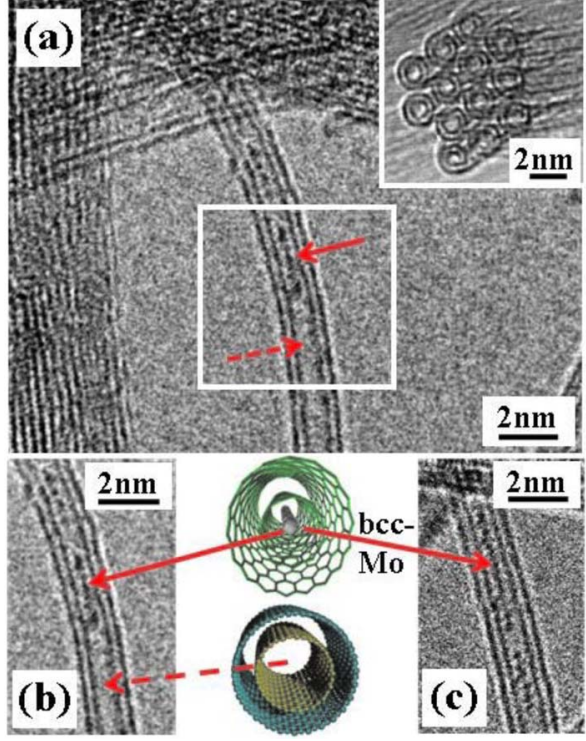

FIG. 1. (Color online) HRTEM images of LMo-DWNTs $[(a)$ and (b) $]$ and HMo-DWNTs (c). Inset shows cross-sectional image.

appear to be arranged in half a cell of bcc-Mo. HRTEM images from the cross sections and side view [inset of Fig. 1(a)] revealed the perfect hexagonal packing structure. The insertion of the Mo atoms inside the hollow cores of DWNTs indicates removal of caps and/or presence of nanowindows in order to let the metal atoms enter the tubules. The opening state of the Mo-doped DWNT can be studied using gas adsorption, giving information on the Mo clusters in the tube spaces and the filling rates of the tube spaces by Mo clusters. HRTEM images of LMo-DWNTs and HMo-DWNTs showed the structures of one-dimensional Mo wires consisting of half a cell of bcc-Mo, which has been recently demonstrated theoretically [Figs. 1(b) and 1(c)]. The dense filling of Mo atoms in HMo-DWNT suggests that the growth of onedimensional clusters of Mo atoms is controllable and depends on the reaction conditions and initial concentrations.

Nitrogen adsorption isotherms at $77 \mathrm{~K}$ for DWNTs, LMo-DWNTs and HMo-DWNTs, shown in Fig. 2(a), were of IUPAC type II with a steep uptake below relative pressure $P / P_{0}=0.05$ and a gradual uptake of nitrogen at the higher $P / P_{0} \cdot{ }^{9}$ Because the adsorption of nitrogen at higher $P / P_{0}$ is associated with the multilayer adsorption on the external surface and marcopores/larger mesopores, the remarkably similar features of these isotherms at higher $P / P_{0}$ indicate that the growth of one-dimensional Mo wires in DWNTs did not significantly change the interparticle voids or the bundle structures. As can be seen in Fig. 2(a) (inset), nitrogen adsorption isotherms for DWNTs, LMo-DWNTs, and HMoDWNTs can be divided as parts I, II, and III, which feature narrow micropore filling steps, indicating the high degree of micropore size uniformity. The subtracting pore effect analysis and the Dubinin-Radushkevich method of the nitrogen adsorption isotherms of LMo-DWNTs, HMo-DWNTs, and DWNTs indicated a decrease of nanoporosities with the growth of one-dimensional Mo wires in DWNTs. ${ }^{9,10}$ The specific surface areas determined with Brunauer-EmmettTeller (BET) method (BET SSA $)^{9}$ and $\alpha_{\text {s }}$-plot method $\left(\alpha_{\mathrm{s}} \text {-plot SSA }\right)^{10}$ of DWNTs, LMo-DWNTs, and HMoDWNTs, and the percentages $\phi$ of micropore volume re-
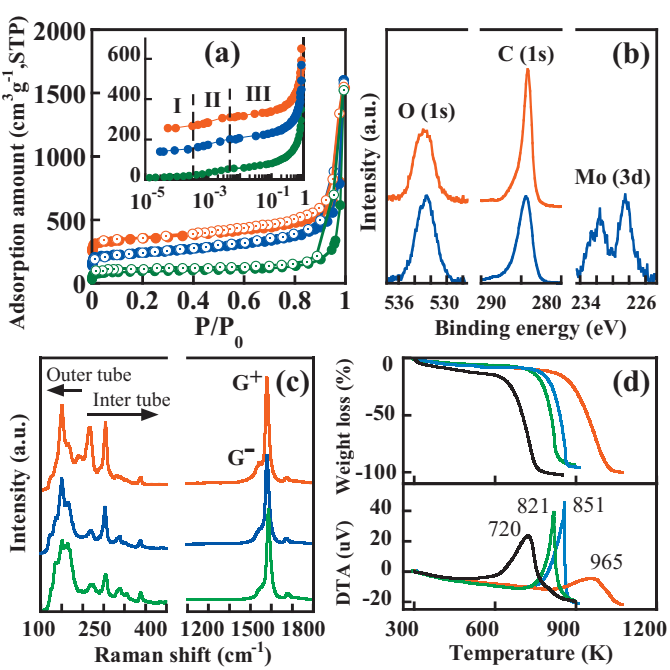

FIG. 2. (Color) (a) Adsorption ( )/desorption (○) isotherms of nitrogen at $77 \mathrm{~K}$. Inset: nitrogen adsorption isotherms at low relative pressures in logarithmic scale. To allow for a better comparison, the adsorption data for LMo-DWNTs and DWNTs were offset vertically by 100 and $200 \mathrm{~cm}^{3}$ STP $g^{-1}$, respectively. (b) Mo $3 d, \mathrm{C} 1 s$, and O $1 s$ XPS spectra. (c) Raman spectra measured using excitation laser energy of $1.96 \mathrm{eV}$. (d) TG/DTA curves (heating rate $5 \mathrm{~K} \mathrm{~min}^{-1}$ in air). Red curves for DWNTs, blue curves for LMo-DWNTs, green curves, HMo-DWNTs, and black curves SWNTs.

duced by Mo doping are presented in Table I. These products have relatively high specific surface areas.

Figure 2(b) shows the Mo $3 d, \mathrm{C} 1 s$, and O $1 s$ XPS spectra of DWNTs and LMo-DWNTs. Distinct peaks located at ca. $284.3 \mathrm{eV}$ for $\mathrm{C} 1 s$ and $232 \mathrm{eV}$ for $\mathrm{O} 1 s$ were observed, respectively, indicating the presence of oxygen in the carbon nanotubes. The incorporation of oxygen in the carbon nanotubes must result from the oxidation purification process used in the sample preparation. The peak positions and peak areas of $\mathrm{O} 1 s$ do not change on doping of Mo, indicating that carbon nanotube states prevented Mo from oxidation, and the Mo atoms are mainly incorporated to the carbon nanotube rather than the molybdenum oxides deposited outside the nanotubes. The LMo-DWNTs revealed a highly reproducible upward shift $(\sim 0.2 \mathrm{eV})$ of the $\mathrm{C} 1 s$ peak, indicating a different state of the double graphene layers. With the growth of Mo atomic clusters in DWNTs, we observed new Mo $3 d_{5 / 2}$ $(228.5 \mathrm{eV})$ and Mo $3 d_{3 / 2}(231.6 \mathrm{eV})$ peaks, attributed to Mo atom and $\mathrm{Mo}_{2} \mathrm{C}$. ${ }^{11}$ Since we could not detect the Mo $3 d$ signals located at 232.6 and $227.8 \mathrm{eV}$, it is clear that the Mo was not oxidized in the tube space of DWNT.

In the Raman spectra of DWNTs, LMo-DWNTs, and HMo-DWNTs [Fig. 2(c)], the features were similar to each other with respect to the peak positions but the peak intensities were quite different. In the radial-breathing-mode (RBM) bands of LMo-DWNTs and HMo-DWNTs, the vibra-

TABLE I. Specific surface areas (SSA), porosity data and the percentages, $\phi$, of micropore volumes reduced by Mo doping.

\begin{tabular}{lccccc}
\hline \hline & $\begin{array}{c}\text { BET SSA } \\
\left(\mathrm{m}^{2} \mathrm{~g}^{-1}\right)\end{array}$ & $\begin{array}{c}\alpha_{\mathrm{s}} \text {-plot SSA } \\
\left(\mathrm{m}^{2} \mathrm{~g}^{-1}\right)\end{array}$ & $\begin{array}{c}\text { Micropore } \\
\text { size } \\
(\mathrm{nm})\end{array}$ & $\begin{array}{c}\text { Micropore } \\
\text { volume } \\
\left(\mathrm{cm}^{3} \mathrm{~g}^{-1}\right)\end{array}$ & $\begin{array}{c}\phi \\
(\%)\end{array}$ \\
\hline HMo-DWNTs & 363 & 320 & 0.68 & 0.12 & 37 \\
LMo-DWNTs & 480 & 580 & 0.68 & 0.17 & 11 \\
DWNTs & 510 & 636 & 0.68 & 0.19 & $\ldots$ \\
\hline \hline
\end{tabular}


tion arising from the inner shells of DWNTs is largely quenched. Because the Mo atoms in HMo-DWNTs are more tightly encapsulated in the tube spaces than in LMo-DWNTs, HMo-DWNTs have a deeper depression of RBM bands corresponding to inner tubes. On the other hand, enhanced Raman peaks from the outer shells of both LMo-DWNTs and HMo-DWNTs at about $167 \mathrm{~cm}^{-1}$ are indicative of the modified electronic structure by the wall-wall interaction established between the inner and outer shells. The higher frequency vibration component $\left(G^{+}\right)$at $1584 \mathrm{~cm}^{-1}$, which is attributed to the vibration along the direction of the nanotube axis, does not change on the Mo doping. ${ }^{12}$ On the contrary, the peak intensity of the circumferential direction vibration $G^{-}$decreases on the Mo doping. Therefore, the Raman spectra of LMo-DWNTs and HMo-DWNTs suggest the presence of charge transfer between the Mo atomic chains and the coaxial tubes, giving rise to a stable hybridization between one-dimensional Mo clusters and carbon nanotube. Thus, the growth technique of one-dimensional Mo atomic chain in the cylindrical empty space of DWNT can control the electronic structure of DWNT through a designed charge transfer.

The thermal stabilities of Mo-encapsulated DWNTs are shown in the TG/DTA curves for LMo-DWNTs, HMoDWNTs, and DWNTs [Fig. 2(d)]. Here TG/DTA chart of SWNTs (HiPco, minimum $85 \mathrm{wt} \%$ ) is shown for comparison. TG/DTA data clearly indicate the thermal stability difference of samples. DWNT gives the highest DTA peak at $965 \mathrm{~K}$, whereas the peak of SWNT is observed at the lowest temperature of $720 \mathrm{~K}$. Mo-doped DWNT samples lose their weight between 720 and $965 \mathrm{~K}$. Consequently, Mo-doped DWNT is more thermally stable than SWNT and less stable than DWNT. As encapsulated Mo should accelerate the gasification of carbon, LMo-DWNT gives a higher DTA peak than HMo-DWNT.

In summary, we showed that with DWNT templating, the growth of one-dimensional clusters of Mo atoms in the cores of DWNTs could be controlled by varying the reaction conditions. Mo atoms self-assembled one-dimensional clusters because of the nanospace confinement of DWNTs, causing $11 \%-37 \%$ reduction of the nanoporosities of DWNTs.
Raman and TG analyses indicated that the characteristic properties of Mo-encapsulated DWNTs resulted from a strong Mo atomic cluster dependence of the electronic structure. In principle, control over the growth of onedimensional atomic clusters as well as the high surface areas allow an adjustment of size-dependent electronic structure to the demands of a wide range of applications.

This research was supported by CLUSTER (the second stage) (YT, HM, TH, YAK, ME) and Grand-in-Aid for Specially Promoted Research (Grant No. 19002007) (TH, YAK, ME) of Ministry of Education, Culture, Sports, Science and Technology, Japan. This work was supported in part by CONACY-México Grants Nos. 56787 (Laboratory for Nanoscience and Nanotechnology research-LINAN), 45772 (MT), 41464 Inter American Collaboration (MT), and 58899 Inter American Collaboration (MT).

${ }^{1}$ M. P. Zach, K. H. Ng, and R. M. Penner, Science 290, 2120 (2000).

${ }^{2}$ B. W. Smith, M. Monthioux, and D. E. Luzzi, Nature (London) 396, 323 (1998).

${ }^{3}$ K. Hirahara, K. Suenaga, S. Bandow, H. Kato, T. Okazaki, H. Shinohara, and S. Iijima, Phys. Rev. Lett. 85, 5384 (2000).

${ }^{4}$ A. Hashimoto, H. Yorimitsu, K. Ajima, K. Suenaga, H. Isobe, J. Miyawaki, M. Yudasaka, S. Iijima, and E. Nakamura, Proc. Natl. Acad. Sci. U.S.A. 101, 8527 (2004).

${ }^{5}$ A. D. Zaikin, D. S. Golubev, A. van Otterlo, and G. T. Zimanyi, Phys. Rev. Lett. 78, 1552 (1997).

${ }^{6}$ H. Muramatsu, T. Hayashi, Y. A. Kim, D. Shimamoto, M. Endo, M. Terrones, and M. S. Dresselhaus, Nano Lett. 8, 237 (2008).

${ }^{7}$ V. Meunier, H. Muramatsu, T. Hayashi, Y. A. Kim, D. Shimamoto, H. Terrones, M. S. Dresselhaus, M. Terrones, M. Endo, and B. G. Sumpter, "Properties of one-dimensional molybdenum nanowires in a confined environment" (unpublished).

${ }^{8}$ M. Endo, H. Muramatsu, T. Hayashi, Y. A. Kim, M. Terrones, and M. S. Dresselhaus, Nature (London) 433, 476 (2005).

${ }^{9}$ S. J. Gregg and K. S. W. Sing, Adsorption, Surface Area and Porosity (Academic, London, 1982).

${ }^{10}$ K. Kaneko, C. Ishii, H. Kanoh, Y. Hanzawa, N. Setoyama, and T. Suzuki, Adv. Colloid Interface Sci. 76, 295 (1998).

${ }^{11}$ T. Xia, Q. Li, X. D. Liu, J. Meng, and X. Q. Cao, J. Phys. Chem. B 110, 2006 (2006)

${ }^{12}$ M. S. Dresselhaus, G. Dresselhause, A. Jorio, A. G. Souza, and R. Saito, Carbon 40, 2043 (2002). 